International Journal of Modern Physics C Vol. 26, No. 12 (2015) 1599001 (12 pages) (C) World Scientific Publishing Company DOI: $10.1142 / \mathrm{S} 0129183115990016$

\title{
Author Index (Volume 26)
}

Abdullah, R., see Alqat$\tan$, Z. N.

Acosta-Elías, J., see Esquivel-Gómez, J.

Acosta-Elias, J., see Esquivel-Gómez, J.

Afshari, E. \& Houreh, N. B., Two dimensional numerical modeling of a membrane humidifier with porous media flow field for PEM fuel cell

Ahmad, E., see Hussain, F.

Ahmad, S. A., see Hussain, $F$.

Ahn, Y.-Y., see Zhang, Z.-Y.

Ai, W., Shi, Z. \& Liu, D., Bifurcation analysis method of nonlinear traffic phenomena

Akhlaghi, H., see Balaj, M.

Alqattan, Z. N. \& Abdullah, R., A hybrid artificial bee colony algorithm for numerical function optimization

Araújo, N. A. M., see da Cruz, J. P.

Arjona-Villicaña, P. D., see Esquivel-Gómez, J.

Aydogmus, F., Chaos in a $4 D$ dissipative nonlinear fermionic model

Ba, D.-C., see Yue, X.-J.

Ba, Y.-S., see Yue, X.-J.

Balaj, M., Akhlaghi, H. \& Roohi, E., Rarefied gas flow behavior in micro/ nanochannels under specified wall heat flux

\author{
10 (2015) 1550109 \\ $6(2015) 1550066$ \\ 12 (2015) 1550142 \\ 6 (2015) 1550061 \\ $1(2015) 1550009$ \\ 1 (2015) 1550009 \\ 9 (2015) 1550096
}

$10(2015) 1550111$

8 (2015) 1550087

$10(2015) 1550109$

6 (2015) 1550070

$12(2015) 1550142$

7 (2015) 1550083

4 (2015) 1550037

4 (2015) 1550037

8 (2015) 1550087
Balderas-Navarro, R. E., see Esquivel-Gómez, J.

Bantang, J. Y., see Pang, J. C. S.

6 (2015) 1550066

10 (2015) 1550112

Bednyakov, A. V. \& Tanyıldızı, Ş. H., $A$ Mathematica package for calculation of oneloop penguins in $\mathrm{FCNC}$ processes

Ben Haddou, N., Ez-Zahraouy, H. \& Benyoussef, A., An adaptive routing scheme in scale-free networks

Benyoussef, A., see Ben Haddou, N.

Benyoussef, A., see Marzoug, R.

Benyoussef, A., see Echab, H.

Bikulov, D., Saratov, A. \& Grachev, E., Prediction of the permeability of proppant packs under load

Binesh, A. R., Mousavi, S. M. \& Kamali, R., Effect of temperature-dependency of Newtonian and non-Newtonian fluid properties on the $d y$ namics of droplet impinging on hot surfaces

Boussinot, F., Monasse, B. \& Susini, J.-F., Reactive programming of simulations in physics

Budinski, L., Fabian, J. \& Stipic, M., Modeling groundwater flow by lattice Boltzmann method in curvilinear coordinates
9 (2015) 1550106

(10) 1550112

4 (2015) 1550042

$12(2015) 1550138$

12 (2015) 1550138

1 (2015) 1550007

9 (2015) 1550100

10 (2015) 1550117

12 (2015) 1550132

2 (2015) 1550013
Scientific

www.worldscientific.com 
Cai, F., Chen, H. \& Shu, Z., Web document ranking via active learning and kernel principal component analysis

Cai, J., see Tan, X.-H.

Cai, M.-J., see Kuang, H.

Cai, Y. Q., see Hussain, F.

Candia, L. I., Carbonetti, J., Garcia, G. D. \& SanchezVarretti, F. O., Topology change due to particle heterogeneity in DLAs

Cao, J., Shi, Z. \& Zhou, J., An extended optimal velocity difference model in a cooperative driving system

Cao, J.-L. \& Shi, Z.-K., $A$ novel lattice traffic flow model on a curved road

Carbonetti, J., see Candia, L. I.

Chai, Z., see Liang, H.

Chang, J.-Y. \& Lai, W.C., Simulation study of traffic accidents on a three-lane highway

Chao, L., Asynchronous error-correcting secure communication scheme based on fractional-order shifting chaotic system

Chen, C., Zhang, H., Wu, Y.-H., Feng, W.-Q. \& Zhang, J., Analysis and optimization of crossimmunity epidemic model on complex networks

Chen, H., see Cai, F.

Chen, J., see Lv, M.

Chen, J., see Dong, Y.

Chen, J. \& Fang, Y., Macroscopic modeling for traffic flow on threelane highways

Chen, M., see Kang, Y.

Chen, P., see $\mathrm{Hu}$, J.

Chen, S., see $\mathrm{Wu}, \mathrm{Q}$.

Chen, S., see Gao, Z.

Chen, X.-L., see Wu, Y.

Cheng, S.-L., see Zhang, G.
4 (2015) 1550041

4 (2015) 1550045

6 (2015) 1550064

1 (2015) 1550009

12 (2015) 1550136

5 (2015) 1550054

11 (2015) 1550121

12 (2015) 1550136

7 (2015) 1550074

3 (2015) 1550027

6 (2015) 1550065

4 (2015) 1550039

4 (2015) 1550041

10 (2015) 1550116

8 (2015) 1550095

11 (2015) 1550127

6 (2015) 1550063

11 (2015) 1550131

4 (2015) 1550044

9 (2015) 1550107

11 (2015) 1550125

6 (2015) 1550062
Curzen, N., see Morisi, R.

da Cruz, J. P., Araújo, N.

A. M., Raischel, F. \& Lind, P. G., A thermostatistical approach to scale-free networks

Da Silva, L. R., see Dos Santos, A. M.

Dai, H., see Yue, X.

Dalmagro, F. \& Jimenez, J., Agent based modeling of the coevolution of hostility and pacifism

De Almeida, M. L., see Dos Santos, A. M.

De Concini, G. \& De Martino, D., Overrelaxed hit-and-run Monte Carlo for the uniform sampling of convex bodies with applications in metabolic network biophysics

De Martino, D., see De Concini, G.

de Moura, F. A. B. F., see Filho, V. L. C.

Deng, W. \& Wang, J., Statistical analysis on multifractal detrended cross-correlation coefficient for return interval by oriented percolation

Deng, Y., Hu, H. \& Liu, L., Feedback control of digital chaotic systems with application to pseudorandom number generator

Deyranlou, A., see Mesri, Y.

Ding, M.-S., Jin, N.-D. \& Gao, Z.-K., Modality transition-based network from multivariate time series for characterizing horizontal oil-water flow patterns

Ding, Z.-J., see Li, Q.-L. 3 (2015) 1550032

Dong, H., see Xuan, Q. 2 (2015) 1550023

Dong, J. B., see Jin, Y. 2 (2015) 1550017

Dong, Q., see Li, W.-J. $\quad 9$ (2015) 1550102
3 (2015) 1550034

1 (2015) 1550011

9 (2015) 1550097

2 (2015) 1550021

9 (2015) 1550098

9 (2015) 1550097

1 (2015) 1550010

$1(2015) 1550010$

12 (2015) 1550133

1 (2015) 1550002

2 (2015) 1550022

4 (2015) 1550038 
Author Index

Dong, Y. \& Chen, J., Finite-time outer synchronization between two complex dynamical networks with on-off coupling

Donini, B., see Morisi, R.

Dorso, C. O., see Frank, G. A.

Dos Santos, A. M., De Almeida, M. L., Mendes, G. A. \& Da Silva, L. R., Generalized scale-free homophilic network

Du, W.-B., see Xu, X.-L.

$\mathrm{Du}, \mathrm{Y} .-\mathrm{J}$. , see $\mathrm{Wu}, \mathrm{Y}$.

Ebrahimi, A. \& Roohi, E., Numerical study of flow patterns and heat transfer in mini twisted oval tubes

Echab, H., Lakouari, N., Ez-Zahraouy, H. \& Benyoussef, A., Cellular automata model simulating traffic car accidents in the on-ramp system

Esfahanian, V., see Ghadyani, $M$.

Esmailian, A., Shahrokhi, M. \& Kanjouri, F., Structural, electronic and magnetic properties of $(N, C)$-codoped $\mathrm{ZnO}$ nanotube: First principles study

Esquivel-Gómez, J., Arjona-Villicaña, P. D. \& Acosta-Elías, J., The impact of local processes and the prohibition of multiple links in the topological properties of directed complex networks

Esquivel-Gómez, J., Balderas-Navarro, R. E., Ugalde, E. \& AcostaElías, J., On the growth of directed complex networks with preferential attachment: Effect upon the prohibition of multiple links

Ez-Zahraouy, H., see Ben Haddou, N.
8 (2015) 1550095

1 (2015) 1550011

1 (2015) 1550005

9 (2015) 1550097

12 (2015) 1550141

11 (2015) 1550125

12 (2015) 1550140

9 (2015) 1550100

1 (2015) 1550006

11 (2015) 1550130

12 (2015) 1550142

6 (2015) 1550066

12 (2015) 1550138
Ez-Zahraouy, H., see Echab, H.

Ez-Zahraouy, H., see Marzoug, R.

Fabian, J., see Budinski, L.

Fang, Y., see Chen, J.

Fang, Z. M., Lv, W., Jiang, L.-X., Xu, Q.-F. \& Song, W.-G., Observation, simulation and optimization of the movement of passengers with baggage in railway station

Feng, W.-Q., see Chen, C.

Filho, V. L. C., Lima, R. P. A., de Moura, F. A. B. F. \& Lyra, M. L., Stability of uniform electronic wavepackets in chains and fullerenes

Frank, G. A. \& Dorso, C. O., Evacuation under limited visibility

Fu, B.-B., Zhang, L., Li, S.-B. \& Li, Y.-X., Survivability of public transit network based on network structure entropy

$\mathrm{Fu}, \mathrm{C}$., see Xuan, Q.

Fu, T., Li, C., Guo, L. \& Wang, Y., How we view competitions between the converging Internet social platforms: Does higher user number mean the final victory?

Fu, Y., see Li, W.-J.

Fujiki, T., see Tanimoto, J.

Funaro, D. \& Kashdan, E., Simulation of electromagnetic scattering with stationary or accelerating targets

9 (2015) 1550100

1 (2015) 1550007

2 (2015) 1550013

11 (2015) 1550127

11 (2015) 1550124

4 (2015) 1550039

12 (2015) 1550133

1 (2015) 1550005

9 (2015) 1550104

2 (2015) 1550023

8 (2015) 1550084

9 (2015) 1550102

7 (2015) 1550072

Gaillard, P. \& Gastineau, M., 18 parameter deformations of the Peregrine breather of order 10 solutions of the NLS equation

Galdamez, R. G., see Ngachin, $\mathrm{M}$.
$2(2015) 1550016$

3 (2015) 1550031 
Gao, C., Zhong, L., Li, X., Zhang, Z. \& Shi, N., Combination methods for identifying influential nodes in networks

Gao, Z., Shi, Y. \& Chen, S., Measures of node centrality in mobile social networks

Gao, Z.-K., see Ding, M.-S.

Garcia, G. D., see Candia, L. I.

Gastineau, M., see Gaillard, P.

Ghadyani, M., Esfahanian, V. \& Taeibi-Rahni, M., The use of shock-detecting sensor to improve the stability of Lattice Boltzmann Model for high Mach number flows

Ghazanfarian, J., Saghatchi, R. \& Gorji-Bandpy, M., Turbulent fluidstructure interaction of water-entry/exit of a rotating circular cylinder using SPH method

Gündüç, S., The role of fanatics in consensus formation

Gokaltun, S., see Ngachin, M.

González-Aguilar, H., see Vargas-Olmos, C.

Gorji-Bandpy, M., see Ghazanfarian, J.

Grachev, E., see Bikulov, D.

Guan, X.-M., see Zhu, Y.-B.

Guo, L., see Fu, T.

Guo, Q., see Tong, X.-L.

Guo, X., see Lv, M.

Guo, Z., see Liang, H.

Hagishima, A., see Tanimoto, J.

Harden, S., see Morisi, R. He, X., Niu, K., He, Z., Lin, J. \& Jiang, Z.-Y., Efficient packet transportation on complex networks with nonuniform node capacity distribution

\section{6 (2015) 1550067}

9 (2015) 1550107

3 (2015) 1550034

12 (2015) 1550136

2 (2015) 1550016

1 (2015) 1550006

8 (2015) 1550088

3 (2015) 1550029

3 (2015) 1550031

8 (2015) 1550093

8 (2015) 1550088

10 (2015) 1550117

6 (2015) 1550069

8 (2015) 1550084

5 (2015) 1550059

10 (2015) 1550116

7 (2015) 1550074

7 (2015) 1550072

1 (2015) 1550011

10 (2015) 1550118
He, Z., see He, X.

He, Z., see Zheng, L.

Herrmann, H. J., see Mendoza, $\mathrm{M}$.

Hong, C., see Xu, X.-L.

Hong, W. \& Wang, J., Fluctuation complexity of agent-based financial time series model by stochastic Potts system

Horváth, D., Maliková, Z. \& Lučkaničová, M., The policy-dependent statistics and induced heterogeneity of the supported agents on the inter and intra company levels

Hou, S., see Wang, L.

Hou, S., see Wei, N.

Houreh, N. B., see Afshari, E.

Hu, H., see Deng, Y.

Hu, J., Li, Z., Zhang, H., Wei, J., You, L. \& Chen, P., Experiment and simulation of the bidirectional pedestrian flow model with overtaking and herding behavior

$\mathrm{Hu}, \mathrm{K}$., see $\mathrm{Li}$, J.

Hu, M.-B., see Jing, X.-L.

Hu, Y., see Zhou, X.

Huang, G., Zhang, B., Ren, R. \& Ren, J., An algorithm to find critical execution paths of software based on complex network

Hussain, F., Cai, Y. Q., Khan, M. J. I., Imran, M., Rashid, M., Ullah, H., Ahmad, E., Kousar, F. \& Ahmad, S. A., Enhanced ferromagnetic properties of $\mathrm{Cu}$ doped two-dimensional GaN monolayer

Ikegaya, N., see Tanimoto, $\mathrm{J}$.

Imran, M., see Hussain, F. Jamaati, M., see Mehri, A. Jana, D., see Nath, P.

Jana, D., see Nath, P.

(Jeff) Ban, X., see Wang, X. Jia, C., see Li, H.-J.
10 (2015) 1550118

8 (2015) 1550090

5 (2015) 1550055

12 (2015) 1550141

11 (2015) 1550123

5 (2015) 1550048

12 (2015) 1550143

5 (2015) 1550056

6 (2015) 1550061

2 (2015) 1550022

11 (2015) 1550131

8 (2015) 1550091

6 (2015) 1550068

2 (2015) 1550018

9 (2015) 1550101

1 (2015) 1550009

7 (2015) 1550072

1 (2015) 1550009

9 (2015) 1550105

10 (2015) 1550115

5 (2015) 1550049

2 (2015) 1550015

4 (2015) 1550043 
Author Index

Jia, J.-J., see Ye, B.

Jia, J.-J., see Ye, B.

Jia, N., see Zhao, C.

Jiang, L.-X., see Fang, Z. $\mathrm{M}$.

Jiang, R., see Li, Q.-L.

Jiang, Z.-Y., see He, X.

Jiang, Z.-Y., see Zhang, S.

Jimenez, J., see Dalmagro, $\mathrm{F}$.

Jin, B., Liao, B., Yuan, N. \& Wang, W., Exploring relationship between human mobility and social ties: Physical distance is not dead

Jin, N.-D., see Ding, M.-S.

Jin, Y., Dong, J. B., Li, X. \& Wu, Y., Kinematical measurement of hydraulic tortuosity of fluid flow in porous media

Jing, X.-L., Ling, X., Long, J., Shi, Q. \& Hu, M.-B., Mean first return time for random walks on weighted networks

Kamali, R., see Binesh, A. $\mathrm{R}$.

Kang, Y., Chen, M., Li, X., You, L. \& Yang, B., Laboratory measurement and interpretation of nonlinear gas flow in shale

Kanjouri, F., see Esmailian, A.

Kashdan, E., see Funaro, D.

Khaleque, A. \& Sen, P. Effect of randomness in logistic maps

Khan, M. J. I., see Hussain, F.

Kong, L.-P., Li, X.-G. \& Lam, W. H. K., Traffic dynamics around weaving section influenced by accident: Cellular automata approach

Kousar, F., see Hussain, F.

Krawczyk, M. J., Communities and classes in symmetric fractals
11 (2015) 1550129

4 (2015) 1550040

1 (2015) 1550004

11 (2015) 1550124

3 (2015) 1550032

10 (2015) 1550118

1 (2015) 1550001

9 (2015) 1550098

1 (2015) 1550008

3 (2015) 1550034

2 (2015) 1550017

6 (2015) 1550068

9 (2015) 1550106

6 (2015) 1550063

11 (2015) 1550130

7 (2015) 1550075

8 (2015) 1550086

$1(2015) 1550009$

3 (2015) 1550026

$1(2015) 1550009$

3 (2015) 1550025
Krawczyk, M. J., Classes of states of discrete systems

Kuang, H., Cai, M.-J., Li, X.-L. \& Song, T., Asymmetric effect on singlefile dense pedestrian flow

Kukida, S., see Tanimoto, J.

Lai, W.-C., see Chang, J.-Y.

Lakouari, N., see Echab, H.

Lam, W. H. K., see Kong, L.-P.

Lanconelli, N., see Morisi, R.

$\mathrm{Li}, \mathrm{C}$, see $\mathrm{Fu}, \mathrm{T}$.

Li, D. \& Xu, H., Testing a simple method for computing directly the bulk modulus by NPT simulation: The case of polydisperse hard sphere solids

Li, H., see Li, X.-F.

Li, H.-J., see Zhang, S.

Li, H.-J., Li, H. \& Jia, C., A novel dynamics combination model reveals the hidden information of community structure

Li, H., see Li, H.-J.

Li, J., Yu, K. \& Hu, K., $A$ novel dynamical community detection algorithm based on weighting scheme Li, J.-R., see Zhao, H.-T.

Li, Q.-L., Jiang, R., Ding, Z.-J., Min, J. \& Wang, B.-H., Effect of vehicles' changing lanes in the Biham-Middleton-Levine traffic flow model

Li, Q., see Liang, H.

Li, S. L., see Yang, K. S.

Li, S.-B., see Fu, B.-B.

Li, W.-J., Xu, Y.-Y., Dong, Q., Zhou, J.-L. \& Fu, Y., TaDb: A timeaware diffusion-based recommender algorithm

Li, X., see Jin, Y.

Li, X.-F., Lu, Z.-M. \& Li, H., Controllability of deterministic complex networks
11 (2015) 1550126

6 (2015) 1550064

7 (2015) 1550072

3 (2015) 1550027

9 (2015) 1550100

3 (2015) 1550026

1 (2015) 1550011

8 (2015) 1550084

5 (2015) 1550057

3 (2015) 1550028

1 (2015) 1550001

4 (2015) 1550043

4 (2015) 1550043

8 (2015) 1550091 7 (2015) 1550079

3 (2015) 1550032

7 (2015) 1550074

2 (2015) 1550020

9 (2015) 1550104

9 (2015) 1550102

2 (2015) 1550017

3 (2015) 1550028 
Li, X.-G., see Xie, D.-F.

Li, X.-G., see Kong, L.-P.

Li, X.-L., see Kuang, H.

Li, X.-P., see Tan, X.-H.

Li, X., see Ying, $\mathrm{S}$.

Li, X., see Kang, Y.

Li, X., see Gao, C.

Li, X.-Y., see Wu, Y.

Li, Y.-L., see Tang, C.-L.

Li, Y., see Wang, J.

Li, Y., see Luo, P.

Li, Y.-X., see Fu, B.-B.

$\mathrm{Li}, \mathrm{Z}$., see $\mathrm{Hu}$, J.

Liang, B., see Yan-Dong, $\mathrm{X}$.

Liang, D., see Wang, L.

Liang, H., Chai, Z., Shi, B., Guo, Z. \& Li, Q., Numerical simulations of immiscible displacement in the cavities via lattice Boltzmann method

Liang, M.-G., see Zhang, $\mathrm{S}$.

Liao, B., see Jin, B.

Lima, F. W. S., Evolution of egoism on semi-directed and undirected BarabásiAlbert networks

Lima, F. W. S., Tax evasion dynamics and nonequilibrium Zaklan model with heterogeneous agents on square lattice

Lima, R. P. A., see Filho, V. L. C.

Lin, J., see $\mathrm{He}, \mathrm{X}$.

Lin, J., see Wang, B.

Lin, M., Zhao, G. \& Wang, G., Recurrence quantification analysis for detecting dynamical changes in earthquake magnitude time series

Lind, P. G., see da Cruz, J. $P$.

Ling, X., see Jing, X.-L.

Liu, D., see Ai, W.

Liu, H., see Sun, D.

Liu, J.-G., see Tong, X.-L. Liu, J.-Y., see Tan, X.-H.

Liu, L., see Deng, Y.

Liu, W.-N., see Zhang, G.

Liu, Y., see Sun, L.

Long, J., see Jing, X.-L.
2 (2015) 1550019

3 (2015) 1550026

6 (2015) 1550064

4 (2015) 1550045

11 (2015) 1550128

6 (2015) 1550063

6 (2015) 1550067

11 (2015) 1550125

1 (2015) 1550003

3 (2015) 1550030

5 (2015) 1550050

9 (2015) 1550104

11 (2015) 1550131

10 (2015) 1550108

12 (2015) 1550143

7 (2015) 1550074

1 (2015) 1550001

1 (2015) 1550008

12 (2015) 1550135

3 (2015) 1550035

12 (2015) 1550133

10 (2015) 1550118

12 (2015) 1550134

7 (2015) 1550077

6 (2015) 1550070

6 (2015) 1550068

10 (2015) 1550111

7 (2015) 1550081

5 (2015) 1550059

4 (2015) 1550045

2 (2015) 1550022

6 (2015) 1550062

7 (2015) 1550080

6 (2015) 1550068
Lu, Y.-J., see Yue, X.-J.

Lu, Z.-M., see Lv, M.

Lu, Z.-M., see Li, X.-F.

Lu, Z.-M., Wu, Z., Luo, H. \& Wang, H.-X., Improved community model for social networks based on social mobility

Lučkaničová, M., see Horváth, D.

Luo, P., Li, Y., Wu, C. \& Zhang, G., Toward costefficient sampling methods

Luo, H., see Lu, Z.-M.

Lv, M., Guo, X., Chen, J., Lu, Z.-M. \& Nie, T., Second-order centrality correlation in scale-free networks

Lv, W., see Fang, Z. M.

Lv-Lin, H., see YanDong, $\mathrm{X}$.

Lyra, M. L., see Filho, V. L. C.

Ma, X., see Xuan, Q.

Ma, X.-P., see Ye, B.

Ma, X.-P., see Ye, B.

Ma, Y. \& Zheng, Z., Extracting principal parameters of complex networks

Maliková, Z., see Horváth, D.

Mandal, P. K., see Nath, P.

Martín del Rey, A. \& Rodríguez Sánchez, G., Reversible elementary cellular automaton with rule number 150 and periodic boundary conditions over $\mathbb{F}_{p}$

Marzoug, R., Ez-Zahraouy, H. \& Benyoussef, A., Simulation study of car accidents at the intersection of two roads in the mixed traffic flow

Mehri, A., Jamaati, M. \& Moradi, M., The effect of imposed temperature difference on thermal conductivity in armchair single-walled carbon nanotube
4 (2015) 1550037

10 (2015) 1550116

3 (2015) 1550028

2 (2015) 1550012

5 (2015) 1550048

5 (2015) 1550050

2 (2015) 1550012

10 (2015) 1550116

11 (2015) 1550124

10 (2015) 1550108

12 (2015) 1550133

2 (2015) 1550023

11 (2015) 1550129

4 (2015) 1550040

9 (2015) 1550103

5 (2015) 1550048

5 (2015) 1550049

11 (2015) 1550120

1 (2015) 1550007

9 (2015) 1550105 
Author Index

Mejía Carlos, M., see Vargas-Olmos, C.

Mendes, G. A., see Dos Santos, A. M.

Mendl, C. B., Matrixvalued quantum lattice Boltzmann method

Mendoza, M., Succi, S. \& Herrmann, H. J., Highorder kinetic relaxation schemes as high-accuracy Poisson solvers

Mesri, Y., Niazmand, H. Deyranlou, A. \& Sadeghi, M. R., Fluid-structure interaction in abdominal aortic aneurysms: Structural and geometrical considerations

Min, J., see Li, Q.-L.

Mohammadipour, O. R. \& Niazmand, H. Numerical simulation of a flat electroosmotic driven flow in the presence of a charged mid-plate

Momeni, M., Linear and nonlinear electromagnetic waves in a magnetized quantum electronpositron plasma

Monasse, B., see Boussinot, F.

Monterola, C., see Pang, J. Z. F.

Moradi, M., see Mehri, A.

Moret, M. A., see Rosa Jr, A. C. P.

Morgan, J., see Morisi, R.

Morisi, R., Donini, B., Lanconelli, N., Rosengarden, J., Morgan, J., Harden, S. \& Curzen, N., Semi-automated scar detection in delayed enhanced cardiac magnetic resonance images

Mousavi, S. M., see Binesh, A. R.

Murguía, J. S., see VargasOlmos, C.
8 (2015) 1550093

9 (2015) 1550097

10 (2015) 1550113

5 (2015) 1550055

4 (2015) 1550038

3 (2015) 1550032

7 (2015) 1550078

5 (2015) 1550058

12 (2015) 1550132

3 (2015) 1550024

9 (2015) 1550105

10 (2015) 1550119

1 (2015) 1550011

$1(2015) 1550011$

9 (2015) 1550106

8 (2015) 1550093
Nath, P., Mandal, P. K. \& Jana, D., KardarParisi-Zhang universality class of a discrete erosion model

Nath, P. \& Jana, D. Observation of nonuniversal scaling exponent in a novel erosion model

$\mathrm{Ng}, \mathrm{K}$. M., see Pang, J. Z. F.

Ngachin, M., Galdamez, R. G., Gokaltun, S. \& Sukop, M. C., Lattice Boltzmann simulation of rising bubble dynamics using an effective buoyancy method

$\mathrm{Ni}, \mathrm{G}$., see $\mathrm{Wu}, \mathrm{Z}$.

Ni, J., see Tong, X.-L.

Niazmand, H., see Mesri, Y.

Niazmand, H., see Mohammadipour, O. R.

Nie, C., see Zhao, H.-T.

Nie, T., see Lv, M.

Niu, K., see He, X.

Noorani, M. S. M., see Vahedi, S.

Norouzi, H., see Omidvar, $P$.

Ohkura, K., see Suzuki, $\mathrm{T}$.

Okazawa, M., see Suzuki, T.

Omidvar, P., Norouzi, H. \& Zarghami, A., Smoothed Particle Hydrodynamics for water wave propagation in a channel

Othman, N. B., see Pang, J. Z. F.

Palombi, F. \& Toti, S., Use of dirichlet distributions and orthogonal projection techniques for the fluctuation analysis of steady-state multivariate birth-death systems

Pang, J. C. S. \& Bantang, J. Y., Hodgkin-Huxley neurons with defective and blocked ion channels
5 (2015) 1550049

10 (2015) 1550115

3 (2015) 1550024

3 (2015) 1550031

7 (2015) 1550076

5 (2015) 1550059

4 (2015) 1550038

7 (2015) 1550078

7 (2015) 1550079

10 (2015) 1550116

10 (2015) 1550118

10 (2015) 1550110

8 (2015) 1550085

11 (2015) 1550122

11 (2015) 1550122

8 (2015) 1550085

3 (2015) 1550024

12 (2015) 1550139

10 (2015) 1550112 
Pang, J. Z. F., Othman, N. B., Ng, K. M. \& Monterola, C., Efficiency and robustness of different bus network designs

Pei, A. Q. \& Wang, J., Graphic analysis and multifractal on percolation-based return interval series

Peng, G., A speed gradient viscous continuum model with the consideration of coupling effect for twolane freeways

Pinsker, F., Optical computing with soliton trains in Bose-Einstein condensates

Qin, X. Y., see Yang, K. S.

Raischel, F., see da Cruz, J. P.

Ramadan, O., Systematic wave-equation finite difference time domain formulations for modeling electromagnetic wave-propagation in general linear and nonlinear dispersive materials

Ramírez-Torres, M. T., see Vargas-Olmos, C.

Rashid, M., see Hussain, F. Ren, J., see Huang, G.

Ren, R., see Huang, G.

Rey, A. M. D. \& Sánchez, G. R., An image encryption algorithm based on $3 D$ cellular automata and chaotic maps

Rodríguez Sánchez, G., see Martín del Rey, A.

Rong, F. \& Shi, B., Incompressible lattice Boltzmann model for axisymmetric flows through porous media

Roohi, E., see Ebrahimi, A.

Roohi, E., see Balaj, M.

Rosa Jr, A. C. P., Vaveliuk, P. \& Moret, M. A., Non-Markovian model for the study of pitting corrosion in a water pipe system
3 (2015) 1550024

12 (2015) 1550137

2 (2015) 1550014

7 (2015) 1550082

2 (2015) 1550020

6 (2015) 1550070

4 (2015) 1550046

8 (2015) 1550093

1 (2015) 1550009

9 (2015) 1550101

9 (2015) 1550101

1 (2015) 1450069

11 (2015) 1550120

4 (2015) 1550036

12 (2015) 1550140

8 (2015) 1550087

10 (2015) 1550119
Rosengarden, J., see Morisi, R.

Rosu, H. C., see VargasOlmos, C.

Sabetghadam, F. \& Soltani, E., Simulation of solid body motion in a Newtonian fluid using a vorticity-based pseudospectral immersed boundary method augmented by the radial basis functions

Sadeghi, M. R., see Mesri, Y.

Saghatchi, R., see Ghazanfarian, J.

Sánchez, G. R., see Rey, A. M. D.

Sanchez-Varretti, F. O., see Candia, L. I.

Saratov, A., see Bikulov, D.

Sen, P., see Khaleque, A.

Shahrokhi, M., see Esmailian, A.

Shang, P., see Shi, W.

Shi, B., see Rong, F.

Shi, B., see Liang, H.

Shi, N., see Gao, C.

Shi, Q., see Jing, X.-L.

Shi, W. \& Shang, P., The multiscale analysis between stock market time series

Shi, Y., see Gao, Z.

Shi, Z., see Ai, W.

Shi, Z., see Cao, J.

Shi, Z.-K., see Cao, J.-L.

Shi, Z.-K., see Zhou, J.

Shi, Z.-K., see Yu, S.-W.

Shokouhmand, H., see Takabi, B.

Shu, Z., see Cai, F.

Soltani, E., see Sabetghadam, F.

Song, T., see Kuang, H.

Song, W.-G., see Fang, Z. $M$.

Song-Yang, L., see YanDong, $\mathrm{X}$.

Stipic, M., see Budinski, L.

Succi, S., see Mendoza, M.

Sukop, M. C., see Ngachin, $\mathrm{M}$.
1 (2015) 1550011

8 (2015) 1550093

5 (2015) 1550053

4 (2015) 1550038

8 (2015) 1550088

1 (2015) 1450069

12 (2015) 1550136

10 (2015) 1550117

8 (2015) 1550086

11 (2015) 1550130

6 (2015) 1550071

4 (2015) 1550036

7 (2015) 1550074

6 (2015) 1550067

6 (2015) 1550068

6 (2015) 1550071

9 (2015) 1550107

10 (2015) 1550111

5 (2015) 1550054

11 (2015) 1550121

8 (2015) 1550092

8 (2015) 1550094

4 (2015) 1550047

4 (2015) 1550041

5 (2015) 1550053

6 (2015) 1550064

11 (2015) 1550124

10 (2015) 1550108

2 (2015) 1550013

5 (2015) 1550055

3 (2015) 1550031 
Author Index

Sun, D., Liu, H., Zhang, G. \& Zhao, M., The new car following model considering vehicle dynamics influence and numerical simulation

Sun, D.-H., see Zhang, G. Sun, G.-L., see Tang, C.-L. Sun, L., Liu, Y., Zeng, Q.A. \& Xiong, F., A novel rumor diffusion model considering the effect of truth in online social media

Susini, J.-F., see Boussinot, F.

Suzuki, T., Okazawa, M. \& Ohkura, K., Smallworld property evaluated by exchanging network topology

Taeibi-Rahni, M., see Ghadyani, M.

Takabi, B. \& Shokouhmand, H., Effects of $\mathrm{Al}_{2} \mathrm{O}_{3}-\mathrm{Cu} /$ water hybrid nanofluid on heat transfer and flow characteristics in turbulent regime

Tan, X.-H., Li, X.-P., Zhang, L.-H., Liu, J.Y. \& Cai, J., Analysis of transient flow and starting pressure gradient of power-law fluid in fractal porous media

Tang, C.-L., Sun, G.-L. \& Li, Y.-L., The structural, elastic and electronic properties of $\mathrm{A}_{2} \mathrm{C}_{2} \quad(\mathrm{~A}=\mathrm{Li}$, $\mathrm{Na}, \mathrm{K}, \mathrm{Rb}$ and $\mathrm{Cs})$ : Firstprinciples calculations

Tanimoto, J., Fujiki, T., Kukida, S., Ikegaya, N. \& Hagishima, A., Acquisition of the field measurement data relating to lane change actions

Tanyıldızı, S. H., see Bednyakov, A. V.

Tong, X.-L., Liu, J.-G., Wang, J.-P., Guo, Q. \& $\mathrm{Ni}$, J., Ranking the spreading ability of nodes in network core
7 (2015) 1550081

6 (2015) 1550062

1 (2015) 1550003

7 (2015) 1550080

$12(2015) 1550132$

11 (2015) 1550122

$1(2015) 1550006$

4 (2015) 1550047

4 (2015) 1550045

1 (2015) 1550003

7 (2015) 1550072

4 (2015) 1550042

5 (2015) 1550059
Toti, S., see Palombi, F.

Troisi, A., Can CA describe collective effects of polluting agents?

Ugalde, E., see EsquivelGómez, J.

Ullah, H., see Hussain, F.

Vahedi, S. \& Noorani, M. S. M., Cluster modified projective synchronization between community networks

Vargas-Olmos, C., Murguía, J. S., RamírezTorres, M. T., Mejía Carlos, M., Rosu, H. C. \& González-Aguilar, H. Two-dimensional DFA scaling analysis applied to encrypted images

12 (2015) 1550139

10 (2015) 1550114

6 (2015) 1550066

1 (2015) 1550009

10 (2015) 1550110

Vaveliuk, $\mathrm{P}$, see Rosa $\mathrm{Jr}$. A. C. P.

Wang, B., see Wei, Q.

Wang, B. \& Lin, J., Numerical prediction on deposition of micro-particulate matter in turbulent channel flows

Wang, B.-H., see Li, Q.-L.

Wang, G., see Lin, M.

Wang, H., see Zheng, Y.

Wang, H.-X., see Lu, Z.-M.

Wang, J., see Hong, W.

Wang, J., see Pei, A. Q.

Wang, J., see Wang, X.

Wang, J., see Zhang, C.

Wang, J., see Zhao, D.

Wang, J., Wu, Y. \& Li, Y., Attack robustness of cascading load model in interdependent networks

Wang, J.-P., see Tong, X.-L.

Wang, J., see Deng, W.

Wang, L., Hou, S. \& Liang, D., First-principles investigations on the phase stability, elastic and thermodynamic properties of $\mathrm{Zr}-$ Al alloys

Wang, L. \& Wang, P., Propagation and stability in software: A complex network perspective
12 (2015) 1550134

3 (2015) 1550032

7 (2015) 1550077

5 (2015) 1550051

2 (2015) 1550012

11 (2015) 1550123

12 (2015) 1550137

2 (2015) 1550015

7 (2015) 1550073

8 (2015) 1550089

3 (2015) 1550030

5 (2015) 1550059

1 (2015) 1550002

12 (2015) 1550143

5 (2015) 1550052 
Wang, P., see Wang, L.

Wang, W., see Jin, B.

Wang, W. \& Zhou, J. G., Lattice Boltzmann method for axisymmetric turbulent flows

Wang, X., see Zhao, D.

Wang, X., Wang, J., Zhang, J. \& (Jeff) Ban, $\mathrm{X}$., Lane-changing model with dynamic consideration of driver's propensity

Wang, Y., see Fu, T.

Wei, J., see $\mathrm{Hu}$, J.

Wei, N., Zhang, X., Zhang, C., Hou, S. \& Zeng, Z., First-principles investigations on the elastic and thermodynamic properties of cubic $\mathrm{ZrO}_{2}$ under high pressure

Wei, Q., Xie, C.-J. \& Wang, B., Synchronization in complex dynamical networks coupled with complex chaotic system

Wu, C., see Luo, P.

Wu, Q. \& Chen, S., Epidemic spreading and immunization in nodeactivity networks

Wu, Y., Du, Y.-J., Li, X.Y. \& Chen, X.-L., Exploring the spiral of silence in adjustable social networks

Wu, Y., see Jin, Y.

Wu, Y., see Zhou, X.

Wu, Y.-H., see Chen, C.

Wu, Y., see Wang, J.

Wu, Z., Xu, Q., Ni, G. \& $\mathrm{Yu}$, G., The study of genetic information flux network properties in genetic algorithms

$\mathrm{Wu}, \mathrm{Z}$., see Lu, Z.-M.

Wu, Z.-H., see Yue, X.-J.

Xie, C.-J., see Wei, Q.

Xie, D.-F., Zhao, X.-M. \& Li, X.-G., Cellular automaton modeling of traffic flow at a crosswalk with push button

Xing, P., see Zhang, C.
5 (2015) 1550052

1 (2015) 1550008

9 (2015) 1550099

8 (2015) 1550089

2 (2015) 1550015

8 (2015) 1550084

11 (2015) 1550131

5 (2015) 1550056

6 (2015) 1550060

5 (2015) 1550050

4 (2015) 1550044

11 (2015) 1550125

2 (2015) 1550017

2 (2015) 1550018

4 (2015) 1550039

3 (2015) 1550030

7 (2015) 1550076

2 (2015) 1550012

4 (2015) 1550037

6 (2015) 1550060

2 (2015) 1550019 7 (2015) 1550073
Xiong, F., see Sun, L.

Xiong, X., see Zhou, X.

$\mathrm{Xu}, \mathrm{H}$., see Li, D.

Xu, J. \& Zhang, Y., Event ambiguity fuels the effective spread of rumors

Xu, Q.-F., see Fang, Z. M.

$\mathrm{Xu}, \mathrm{Q}$., see $\mathrm{Wu}, \mathrm{Z}$.

Xu, X.-L., Du, W.-B. \& Hong, C., Cascade defense via routing in complex networks

$\mathrm{Xu}$, Y.-Y., see Li, W.-J.

Xuan, Q., Ma, X., Fu, C., Dong, H., Zhang, G. \& $\mathrm{Yu}$, L., Heterogeneous multidimensional scaling for complex networks

Yan-Dong, X., SongYang, L., Lv-Lin, H. \& Liang, B., Mitigation of malicious attacks on network observation

Yang, B., see Kang, Y.

Yang, K. S., Li, S. L., Zhang, J., Zeng, Z., Qin, X. Y. \& Zhou, X. M., Phase transition of iron doped $\mathrm{MgO}$ under high pressure by firstprinciples study

Ye, B., Jia, J.-J.,Zuo, K.-W.\& Ma, X.-P., Can the structural robustness of complex networks be enhanced by interconnection?

Ye, B., Zuo, K.-W., Jia, J.J. \& Ma, X.-P., The epidemic cost of interconnected networks

Ying, S., Li, X. \& Zhong, $\mathrm{X}$, Initial value sensitivity of the Chinese stock market and its relationship with the investment psychology

You, L., see $\mathrm{Hu}$, J.

You, L., see Kang, Y.

$\mathrm{Yu}, \mathrm{G}$., see $\mathrm{Wu}, \mathrm{Z}$.

$\mathrm{Yu}, \mathrm{K}$., see Li, J.

Yu, L., see Xuan, Q.

Yu, S.-W. \& Shi, Z.-K., An improved car-following model with two preceding cars' average speed
7 (2015) 1550080

2 (2015) 1550018

5 (2015) 1550057

3 (2015) 1550033

11 (2015) 1550124

7 (2015) 1550076

12 (2015) 1550141

9 (2015) 1550102

2 (2015) 1550023

10 (2015) 1550108

6 (2015) 1550063

2 (2015) 1550020

4 (2015) 1550040

11 (2015) 1550129

11 (2015) 1550128

11 (2015) 1550131

6 (2015) 1550063

7 (2015) 1550076

8 (2015) 1550091

2 (2015) 1550023

8 (2015) 1550094 
Author Index

Yuan, J., see Yue, X.

Yuan, N., see Jin, B.

Yue, X., Dai, H. \& Yuan,

J., Stochastic bifurcation in a model of love with colored noise

Yue, X.-J., Wu, Z.-H., Ba, Y.-S., Lu, Y.-J., Zhu, Z.-P. \& Ba, D.-C., Lattice-Boltzmann simulation for pressure driven microscale gas flows in transition regime

Zarghami, A., see Omidvar, $\mathrm{P}$.

Zeng, Q.-A., see Sun, L.

Zeng, Z., see Yang, K. S.

Zeng, Z., see Wei, N.

Zhang, B., see Huang, G.

Zhang, C., see Wei, N

Zhang, C., Xing, P. \& Wang, J., Quality effort decision in service supply chain with quality preference based on quantum game

Zhang, G., see Xuan, Q.

Zhang, G., see Luo, P.

Zhang, G., see Sun, D.

Zhang, G., Sun, D.-H., Zhao, M., Liu, W.-N \& Cheng, S.-L., Analysis of average density difference effect in a new twolane lattice model

Zhang, H., see $\mathrm{Hu}$, J.

Zhang, H., see Chen, C.

Zhang, J., see Wang, X.

Zhang, J., see Yang, K. S

Zhang, J., see Chen, C.

Zhang, L., see Fu, B.-B.

Zhang, L.-H., see Tan, X.-H.

Zhang, S., Liang, M.-G., Jiang, Z.-Y. \& Li, H.-J., Improved efficient static weighted routing strategy on two-layer complex networks

Zhang, X., see Wei, N.

Zhang, X., see Zhao, D.

Zhang, X.-J., see Zhu, Y.-B.

Zhang, Y., see $\mathrm{Xu}, \mathrm{J}$.

Zhang, Z., see Gao, C.
2 (2015) 1550021

1 (2015) 1550008

2 (2015) 1550021

4 (2015) 1550037

8 (2015) 1550085

7 (2015) 1550080

2 (2015) 1550020

5 (2015) 1550056

9 (2015) 1550101

5 (2015) 1550056

7 (2015) 1550073

2 (2015) 1550023

5 (2015) 1550050

7 (2015) 1550081

6 (2015) 1550062

11 (2015) 1550131

4 (2015) 1550039

2 (2015) 1550015

2 (2015) 1550020

4 (2015) 1550039

9 (2015) 1550104

4 (2015) 1550045

1 (2015) 1550001

5 (2015) 1550056

8 (2015) 1550089

6 (2015) 1550069

3 (2015) 1550033

6 (2015) 1550067
Zhang, Z.-Y. \& Ahn, Y.Y., Community detection in bipartite networks using weighted symmetric binary matrix factorization

Zhao, C. \& Jia, N., The impact of iterated games on traffic flow at noncontrolled intersections

Zhao, D., Wang, J., Zhang, X. \& Wang, X., A Cellular Automata occupant evacuation model considering gathering behavior

Zhao, G., see Lin, M.

Zhao, H.-T., Li, J.-R. \& Nie, C., Cellular automaton models for traffic flow considering opposite driving of an emergency vehicle

Zhao, M., see Zhang, G.

Zhao, M., see Sun, D.

Zhao, X.-M., see Xie, D.-F.

Zheng, L. \& He, Z., A new car following model from the perspective of visual imaging

Zheng, Z., see Ma, Y.

Zheng, Y. \& Wang, H., Chaotic burst synchronization in a two-smallworld-layer neuronal network

Zhong, L., see Gao, C.

Zhong, $\mathrm{X}$., see Ying, $\mathrm{S}$.

Zhou, J. G., see Wang, W.

Zhou, J., see Cao, J.

Zhou, J. \& Shi, Z.-K., Lattice hydrodynamic model for bidirectional pedestrian flow with the consideration of pedestrian density difference Zhou, J.-L., see Li, W.-J.

Zhou, X. M., see Yang, K. S.

Zhou, X., Hu, Y., Wu, Y. \& Xiong, X., Influence analysis of information erupted on social networks based on SIR model
9 (2015) 1550096

1 (2015) 1550004

8 (2015) 1550089 7 (2015) 1550077

7 (2015) 1550079

6 (2015) 1550062

7 (2015) 1550081

2 (2015) 1550019

8 (2015) 1550090

9 (2015) 1550103

5 (2015) 1550051

6 (2015) 1550067

11 (2015) 1550128

9 (2015) 1550099

5 (2015) 1550054

8 (2015) 1550092

9 (2015) 1550102

2 (2015) 1550020

2 (2015) 1550018 
Zhu, Y.-B., Guan, X.-M. \& Zhang, X.-J., Heterogeneous delivering capability promotes traffic efficiency in complex networks
$6(2015) 1550069$

Zhu, Z.-P., see Yue, X.-J. 4 (2015) 1550037

Zuo, K.-W., see Ye, B. 11 (2015) 1550129

Zuo, K.-W., see Ye, B. 4 (2015) 1550040 